Case report

\title{
Rasburicase induced severe hemolysis and methemoglobinemia in a Caucasian patient complicated by acute renal failure and ARDS
}

\author{
Yonas Raru ${ }^{\mathrm{a}, *}$, Mahmoud Abouzid $^{\mathrm{a}}$, Julia Parsons ${ }^{\mathrm{a}}$, Fuad Zeid ${ }^{\mathrm{b}}$ \\ ${ }^{a}$ Internal Medicine Resident, Marshall University School of Medicine, Huntington, WV, USA \\ ${ }^{\mathrm{b}}$ Pulmonary and Critical Care Medicine, Marshall University School of Medicine, Huntington, WV, USA
}

\section{A B S T R A C T}

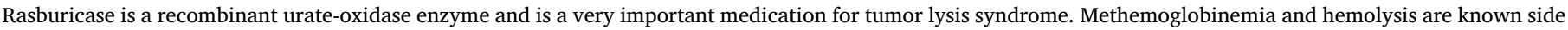

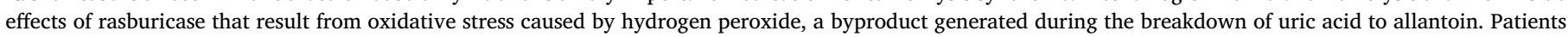

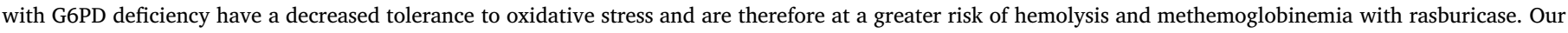

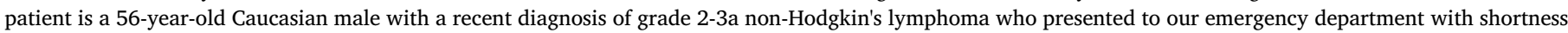

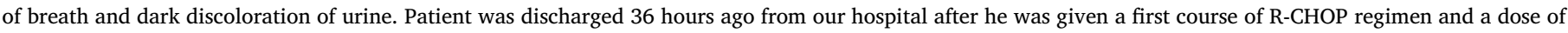

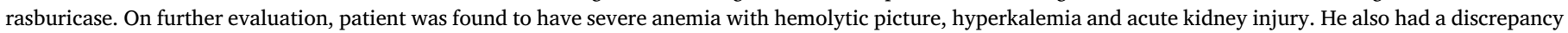

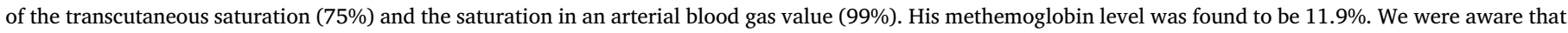

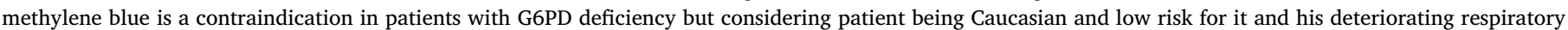

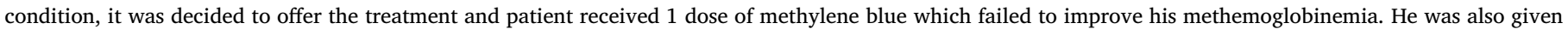

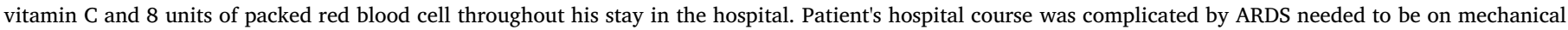

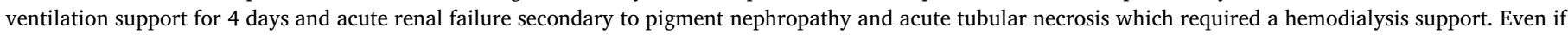

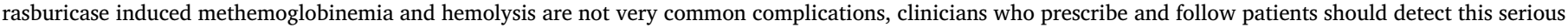

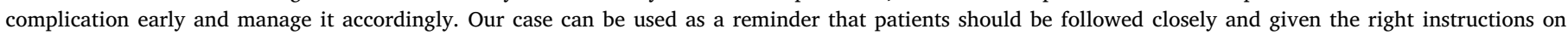

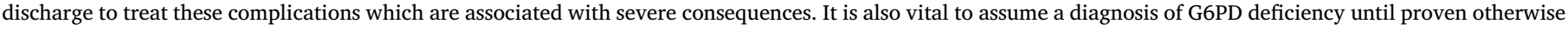

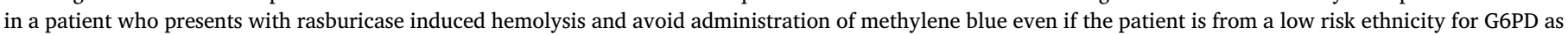
in our patient.

\section{Introduction}

Tumor lysis syndrome (TLS) is an oncologic emergency caused by massive tumor cell lysis with the release of large amounts of potassium, phosphate, and nucleic acids into systemic circulation. Catabolism of these nucleic acids into uric acid leads to hyperuricemia [1,2,11]. TLS most commonly occurs after the initiation of cytotoxic therapy in patients with high-grade lymphomas and acute lymphoblastic leukemia. However, TLS can occur spontaneously and with other tumor types with high proliferative rates, large tumor burden, or high sensitivity to cytotoxic therapy [11]. Rasburicase is a recombinant urate-oxidase enzyme which converts uric acid into allantoin, an inactive, soluble metabolite of uric acid [4]. Previous studies revealed that rasburicase reduces serum urate levels faster and with less acute kidney injury compared to allopurinol, so it is commonly used in patients with clinical TLS or in patients who are at high risk for TLS as is the case with high-grade lymphomas and acute lymphoblastic leukemia [1,5,6]. Methemoglobinemia and hemolysis are known side effects of rasburicase that result from oxidative stress caused by hydrogen peroxide, a byproduct generated during the breakdown of uric acid to allantoin. Patients with G6PD deficiency have a decreased tolerance to oxidative stress and are therefore at a greater risk of hemolysis and methemoglobinemia with rasburicase [6,8]. We present a Caucasian patient of north European descent with rasburicase induced severe hemolytic anemia and methemoglobinemia with a newly diagnosed glucose 6 phosphate dehydrogenase deficiency ().

\section{Case report}

A 56-year-old Caucasian male of north American descent with recent diagnosis of grade 3 a follicular lymphoma, tobacco and marijuana use disorder presented to the emergency department with shortness of breath and hematuria. He was diagnosed with grade 3 follicular lymphoma two weeks prior to this admission when he presented to our hospital with abdominal pain and back pain associated with six-week duration of abdominal distention, decreased appetite and 14-kilogram

\footnotetext{
* Corresponding author.

E-mail address: raru@marshall.edu (Y. Raru).
} 


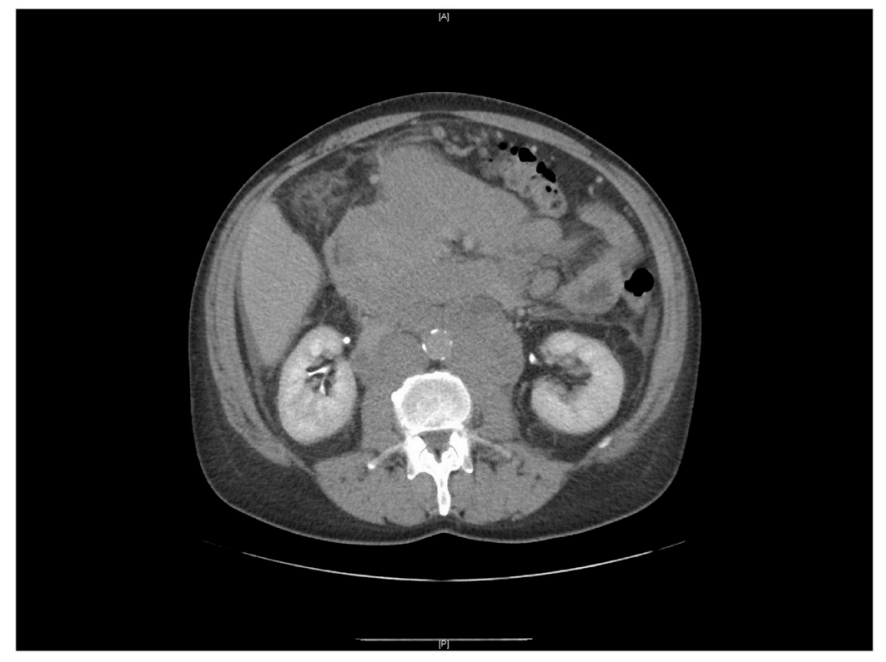

Fig. 1. Laboratory values at admission and 36 hours before (Last discharge from hospital).

weight loss. He underwent CT scan of abdomen at that time showing bulky retroperitoneal and mesenteric lymphadenopathy consistent with lymphoma and large volume ascites (Fig. 1). CT chest was also performed showing normal lung parenchyma with no involvement. The patient underwent CT guided core biopsy showing WHO grade $3 \mathrm{a}$ follicular lymphoma. Oncology was consulted, and he underwent a bone marrow biopsy showing normocellular bone marrow with no involvement by lymphoma. Peripheral blood revealed slight normocytic anemia but was otherwise normal. At the time of the first admission, hemoglobin was $13 \mathrm{~g} / \mathrm{dl}$, white blood cell count was 10300 per cubic millimeter, uric acid $5.7 \mathrm{mg} / \mathrm{dl}$, LDH $662 \mathrm{u} / \mathrm{l}$, bilirubin $1.1 \mathrm{mg} / \mathrm{dl}$ ad alkaline phosphatase was 201U/L. Electrolytes were within the normal range. Due to the high-grade nature of the lymphoma, the patient was admitted to the hospital to start chemotherapy. After appropriate counseling, he received Rituximab, cyclophosphamide, doxorubicin, vincristine, and prednisone. Twenty-four hours after receiving the first dose of chemotherapy, he had a significant increase in uric acid and phosphorus levels raising the concern for possible tumor lysis syndrome. He was given one dose of Rasburicase and his uric acid and phosphorus normalized. He was asymptomatic and was discharged home.

Around 12 hours after the administration of Rasburicase, he began to experience shortness of breath, dizziness, and noticed that his urine became dark. He fell at home and injured his right arm but sustained no head trauma. He then noticed bloody urine, increased weakness and shortness of breath and he came to the emergency room (ER) for evaluation.

On arrival, patient denied any chest pain, cough, fever, chills, nausea or vomiting. He was alert and oriented, tachycardic and in mild respiratory distress. Oxygen saturation was around $75 \%$. He had mild abdominal distension. There was decreased air entry in the right lower posterior chest.

His laboratory findings are as follows:

\begin{tabular}{llll}
\hline & Normal & At admission & 36 hours before \\
\hline WBC (K/cubic mm) & $4-11$ & 45.4 & 15.1 \\
Hemoglobin (g/dl) & $13-15$ & 7.9 & 13.4 \\
Potassium (meq/l) & $3.5-5$ & 7.1 & 4.8 \\
Creatinine (mg/dl) & $0.6-1.1$ & 1.11 & 0.8 \\
Phosphorous (mg/dl) & $2.5-4.5$ & 3.9 & 3.2 \\
LDH (u/l) & $87-241$ & 3800 & 740 \\
Bilirubin (mg/dl) & $0.2-1$ & 7 & 1 \\
Haptoglobin & $30-200$ & 26 & - \\
\hline
\end{tabular}

At initial evaluation, he was in mild distress, but his transcutaneous saturation was around $75 \%$. He was placed on bi-valve positive air way pressure from the emergency room. CT of the chest with pulmonary embolism protocol was negative and there was no abnormal pulmonary infiltrate. Arterial blood gas was ordered, and it showed a PH of 7.51, PCO2 of 35 and PO2 of 565 and oxygen saturation of $100 \%$. The discrepancy of the transcutaneous saturation and the arterial blood gas value lead us for a possible diagnosis of methemoglobinemia secondary to Rasburicase and methemoglobin and carboxyhemoglobin levels were ordered. Laboratory values with time after admission is shown in Table 1.

The methemoglobin level was found to be $11.9 \%$ and his carboxyhemoglobin level was 7.5\%. Rasburicase induced methemoglobinemia with severe hemolytic anemia, hyperkalemia and acute kidney injury was diagnosed. Since Rasburicase mostly causes hemolysis in patients with glucose 6 phosphate dehydrogenase deficiency (G6PD), quantitative test for it was sent even if our patient is a Caucasian male of north European descent with less probability of abnormal test. Hemoglobin was followed regularly and patient was placed on supportive management including steroids. But his respiratory condition worsened, and it was decided to start the patient with methylene blue. We were aware that methylene blue is contraindicated in patients with G6PD deficiency but considering low risk race for G6PD deficiency and his deteriorating respiratory condition, it was decided to offer the treatment for the patient.

Patient was counselled about the situation and he decided he wanted the treatment and he was given one dose of $1 \mathrm{mg} / \mathrm{kg}$ of methylene blue and he was also started with vitamin $C$ and continued with IV fluid. Methemoglobin level was remeasured 2 hours after administration of the methylene blue, but patient didn't show any clinical or biochemical improvement. He was continued with supportive care and blood transfusion. His methemoglobin level gradually came down to $1.9 \%$ and carboxyhemoglobin level to 4\%. The G6PD level came back slightly lower than normal which makes it an abnormal test since the expected result in acute hemolysis is normal to higher than normal

Table 1

Laboratory values with time after admission.

\begin{tabular}{|c|c|c|c|c|c|c|c|c|}
\hline & Normal & Before Rasburicase & Admission & 12 hours & 24 hours & 36 hours & 48 hours & 60 hours \\
\hline WBC, K/cubic mm & $4-11$ & 15.1 & 45.4 & 46.6 & 37.5 & 36.2 & 33.6 & 25.8 \\
\hline $\mathrm{Hgb}, \mathrm{g} / \mathrm{dl}$ & $13-15$ & 13.4 & 7.9 & 6.1 & 7.1 & 7.5 & 7.6 & 7.3 \\
\hline Potassium, meq/1 & $3.5-5$ & 4.4 & 7.1 & 5.4 & 5.2 & 4.2 & 3.7 & 3.5 \\
\hline Creatinine, mg/dl & $0.6-1.1$ & 0.74 & 1.1 & 1.32 & 1.60 & 1.72 & 2.26 & 3.82 \\
\hline Phosphorous, mg/dl & $2.5-4.5$ & 3.6 & 3.2 & 3.9 & 5.2 & 5.5 & 4.9 & 4 \\
\hline $\mathrm{LDH}, \mathrm{u} / \mathrm{l}$ & $87-241$ & 743 & 3800 & 5103 & 5650 & 5150 & 2340 & 1066 \\
\hline Bilirubin, mg/dl & $0.2-1$ & 1.2 & 7 & 6.3 & 4 & 2.7 & 1.3 & 1.1 \\
\hline Uric acid, mg/dl & $3.5-7.2$ & 7.1 & 1.2 & 3 & 3 & 3.1 & 3.1 & 4.1 \\
\hline Haptoglobin, mg/dl & $30-200$ & - & 26 & 26 & 27 & 26 & 100 & 165 \\
\hline Methemoglobin, \% & $0-1.5 \%$ & - & 11.1 & 11.9 & 6.9 & 3.1 & 1.9 & 1.8 \\
\hline Carbon monoxide, \% & $0-3 \%$ & - & 7.5 & 6.9 & 5.8 & 4.6 & 4.1 & 3.9 \\
\hline
\end{tabular}


values. Patient subsequently developed acute respiratory distress syndrome because of repeated blood transfusions due to severe anemia secondary to rasburicase induced hemolysis and IV fluid administration and he was intubated, and mechanical ventilation support was given. He needed to stay on mechanical ventilation for 4 days and was extubated. The extent of the hemolysis due to rasburicase was so severe that patient needed around 8 units of blood and he developed acute renal failure due to pigment nephropathy and acute tubular necrosis for which he was placed on hemodialysis throughout his stay in the hospital.

\section{Discussion}

Rasburicase is a recombinant urate-oxidase enzyme, which converts uric acid to allantoin which is an inactive and soluble metabolite of uric acid. It does not inhibit the formation of uric acid [1]. Rasburicase has black box warnings as it may cause serious and fatal hypersensitivity reactions including anaphylaxis, hemolysis in patients with glucose-6phosphate dehydrogenase (G6PD) deficiency, and methemoglobinemia [5]. Clinicians who prescribe or follow patients who are given rasburicase should be able to recognize and promptly manage hemolysis and methemoglobinemia associated with rasburicase as it is a serious complication with a severe consequence. Our patient had a discrepancy in the transcutaneous saturation and the saturation in the arterial blood gas value which tipped us for a possibility of methemoglobinemia secondary to Rasburicase.

He also had a drop of around $5.5 \mathrm{mg} / \mathrm{dl}$ of hemoglobin in 36 hours which also gave us another clue that the patient has a complication from the rasburicase. Screening patients at high risk of glucose-6phosphate dehydrogenase (G6PD) deficiency, individuals of African and Mediterranean descent, is recommended prior to initiation of rasburicase even if it is not done frequently in practice $[2,3,7,9,10]$. We were aware that methylene blue is contraindicated in patients with G6PD deficiency but considering patient being Caucasian of European descent and low risk for it and his deteriorating respiratory condition, it was decided to offer the treatment for him. On the other hand, it is also suggested that a high index of suspicion of G6PD deficiency is also crucial in a patient who develops methemoglobinemia or hemolytic anemia following rasburicase therapy, irrespective of ethnic origin $[1,2,10,12,13]$.

G6PD deficiency is associated with NADPH deficiency, and methylene blue is dependent on NADPH to reduce methemoglobin to hemoglobin. This renders methylene blue ineffective in G6PD-deficient patients, and it may aggravate methemoglobinemia at high doses by oxidizing hemoglobin $[4,12,14]$. Methemoglobin is generated when hemoglobin iron is oxidized from the ferrous (Fe2p) to the ferric (Fe3p) state. Administered methylene blue is converted to leukomethylene blue by the NADPH-dependent methemoglobin reductase system, which then transfers an electron to ferric iron reducing it to ferrous iron [3]. In retrospect, it would have been wiser not to give our patient the methylene blue considering his presentation with severe hemolysis and the low G6PD level that was demonstrated in the laboratory result which came back after 3 days even if patient was actively in hemolysis when the blood sample was taken. Most previously reported cases of rasburicase induced hemolysis and methemoglobinemia with G6PD deficiency were males just like our patient which could be explained by the X-linked mode of inheritance. But the condition was also reported in females [9]. We also used ascorbic acid on our patient. Ascorbic acid has an antioxidant effect not dependent on NADPH and is a safe and effective treatment for methemoglobinemia especially for patients with G6PD deficiency [1,4,6]. Rasburicase induced methemoglobinemia could develop as early as 2 hours after drug administration but our patient started to have symptoms 12 hours after rasburicase was given. This has a very important implication when patients are treated with rasburicase in an outpatient setting [2,3]. It is suggested to observe patients for at least 6 hours and then give proper instructions for the next 24 hours. Our patient was advised about the risks of rasburicase and was observed for about 6 hours but stayed at home 24 hours more after he started to have the symptoms which further complicated his condition.

Previous literature has shown that there is no apparent relationship between the dose of rasburicase administered and the peak methemoglobin level or the lowest hemoglobin level [3,8]. Most of the cases in literature responded for treatment within 3-7 days and our patient has responded for the treatment in that time range but he has more complications including ARDS and requirement for mechanical ventilation support, multiple transfusions and acute renal failure needing hemodialysis. Even if rasburicase induced methemoglobinemia and hemolysis are not very common complications, clinicians who prescribe and follow patients should detect this serious complication early and manage it accordingly. Our case can be used as a reminder that patients should be followed and given the right instructions on discharge to treat this complication which is associated with severe consequences.

\section{Conclusion}

Rasburicase is a recombinant urate-oxidase enzyme and is a very important medication for tumor lysis syndrome. It has black box warnings as it may cause serious and fatal hypersensitivity reactions including anaphylaxis, hemolysis in patients with glucose-6-phosphate dehydrogenase (G6PD) deficiency, and methemoglobinemia. Even if rasburicase induced methemoglobinemia and hemolysis are not very common complications, clinicians who prescribe and follow patients should detect this serious complication early and manage it accordingly. Our case can be used as a reminder that patients should be followed closely and given the right instructions on discharge to treat these complications which are associated with severe consequences. It is also vital to assume a diagnosis of G6PD deficiency until proven otherwise in a patient who presents with rasburicase induced hemolysis and avoid administration of methylene blue even if the patient is from a low risk ethnicity like Caucasian as in our patient.

\section{Conflict of interest}

The authors have no conflict of interest.

\section{References}

[1] C.Y. Cheah, T.,E. Lew, J.,F. Seymour, K. Burbury, Rasburicase causing severe oxidative hemolysis and methemoglobinemia in a patient with previously unrecognized glucose-6-phosphate dehydrogenase deficiency, Acta Haematol. 130 (2013) 254-259.

[2] A. Oluwasanjo, et al., Rasburicase-induced methaemoglobinaemia and G6PD deficiency in an unusual suspect, Br. J. Haematol. 170 (5) (Sep 2015) 595.

[3] M.B. Sonbol, H. Yadav, R. Vaidya, V. Rana, T.E. Witzig, Methemoglobinemia and hemolysis in a patient with G6PD deficiency treated with rasburicase, Am. J. Hematol. 88 (2013) 152-154.

[4] G.B. Sherwood, et al., Rasburicase-induced methemoglobinemia: case report, literature review, and proposed treatment algorithm, Clin. Case Rep. 4 (4) (Apr 2016) 315-319.

[5] Elitek (rasburicase), US Food and Drug Administration, (2013).

[6] L.A. Browning, J.A. Kruse, Hemolysis and methemoglobinemia secondary to rasburicase administration, Ann. Pharmacother. 39 (2005) 1932-1935.

[7] P. Bhat, I. Sisler, A.B. Collier, Exchange transfusion as treatment for rasburicase induced methemoglobinemia in a glucose-6-phosphate dehydrogenase deficient patient, Pediatr. Blood Cancer 51 (2008) 568.

[8] M.H. Bucklin, C.M. Groth, Mortality following rasburicase-induced methemoglobinemia, Ann. Pharmacother. 47 (2013) 1353-1358. 
[9] D.A. Roberts, J.A. Freed, Rasburicase-induced methemoglobinemia in two African-American female patients: an under-recognized and continued problem, Eur. J. Haematol. 94 (2014) 83-85.

[10] U. Ibrahim, A. Saqib, F. Mohammad, J.P. Atallah, M. Odaimi, Rasburicase-induced methemoglobinemia: the eyes do not see what the mind does not know, J. Oncol. Pharm. Pract. 24 (4) (2018 Jun) 309-313.

[11] S.C. Howard, D.P. Jones, C.-H. Pui, The tumor lysis syndrome, N. Engl. J. Med. 364 (19) (2011) 1844-1854.
[12] A. Rehman, M. Shehadeh, D. Khirfan, A. Jones, Severe acute hemolytic anemia associated with severe methemoglobinemia in a G6PD-deficient man, BMJ Case Rep. (2018), https://doi.org/10.1136/bcr-2017-223369.

[13] M. Younis, L. Derbas, S.M. Eikermann, et al., Not the typical pneumonia: an unusual case of rasburicase-induced methomoglobinemia, Cureus 10 (8) (August 01, 2018) e3084, , https://doi.org/10.7759/cureus.3084.

[14] D. Ferguson, et al., Rasburicase-induced hemolytic anemia in previously undiagnosed G6PD deficiency, Blood 132 (6) (9 Aug 2018) 673. 